\title{
The Effects of Cardioversion on the Prothrombotic Markers and CRP in the Atrial Fibrillation
}

\author{
Gökhan Özmen², Mehmet Demir ${ }^{1^{*}}$ and Alparslan Birdane ${ }^{3}$ \\ ${ }^{1}$ Bursa Yüksek Ihtisas Education and Research Hospital, Cardiology Depertment, Bursa/Turkey \\ ${ }^{2}$ Bursa Yüksek Ihtisas Education and Research Hospital, Cardiology Depertment, Bursa/Turkey
} ${ }^{3}$ Osmangazi University, Cardiology Depertment, Eskişehir /Turkey

\section{Background}

Atrial fibrillation (AF) is the most commonly encountered cardiac arrhythmia in clinical practice. There are increasing evidences indicating that $\mathrm{AF}$ is associated with inflamation, abnormal hemostasis, fibrinolysis, thrombocytic and endothelial functions as well as creating a prothrombot.

\section{Introduction}

Atrial fibrillation (AF) is the most commonly encountered cardiac arrhythmia in clinical practice, its prevalence has gradually increased in the last few decades. It is an important healthcare issue since it increases cardiovascular morbidity and mortality. Although maintaining the rate of ventricular response in an acceptable range is an adequate therapeutic approach for certain patients, restoration and maintenance of Sinus Rhythm (SR) is the preferred treatment approach for most patients. The therapeutic approach of the restoration of sinus rhythm has favorable effects such as prevention of electrical and structural remodeling in the atrium, improvement of hemodynamic status, recovering the symptoms and better quality of life [1-4].

Although antiarrhythmic medications are used for the maintenance of SR, a majority of the patients redeveloped AF $[1,2]$. It is generally believed that the recurrence of AF is related to advanced age, atrial enlargement, and prolonged arrhythmia. Arrhythmia becomes more troublesome over time to be treated by physicians because of its tendency to be constant. Increasing evidences suggest the effect of inflammation in genetics and permanence of $\mathrm{AF}[5,6]$. The association of AF with inflammation demonstrated by C-Reactive Protein (CRP) values has attracted many researchers' attention. In recent years, many studies have researched the association between basal CRP levels before $\mathrm{CV}$ and AF recurrence after CV. Even though some of these studies indicated a correlation $[7,8,9]$, the other trials did not confirm such correlation $[10,11]$.

There are increasing evidences indicating that $\mathrm{AF}$ is associated with abnormal hemostasis, fibrinolysis, thrombocytic and endothelial functions as well as creating a prothrombotic and hypercoagulable state $[10,12]$. This prothrombotic state is related to thrombus formation in the left atrium and spontaneous echo contrast [13]. The hypercoagulable state can interact additively with echocardiographic and clinical risk factors for thromboembolism [14]. There is an obvious relationship between thrombogenesis and inflammation. In a study, it was emphasized an association between fibrin D-dimer and CRP, which might be related to ischemic heart disease. There are also studies reporting elevated CRP levels reflecting inflammation and increasing $\mathrm{AF}$ recurrences $[6,8]$.

Our study was conducted to research the association of $\mathrm{AF}$ recurrence with CRP and prothrombotic markers, which were analyzed before $\mathrm{CV}$ and at the visits one day, one month, 3 months and 6 months later. So that, it was ascertained the relationship between CRP and AF recurrence and whether hypercoagulable state of the patients who had higher levels of prothrombotic markers prior to $\mathrm{CV}$ in comparison with the individuals with SR was changed after CV.

\section{Materials and Method}

The study included 139 patients. CV was indicated in 89 patients with atrial fibrillation (mean age was $63,33 \pm 9.14$ ); these patients were performed the procedure and followed throughout six months For electrical CV, a Nihon Kohden defibrillator device with biphasic and monophasic properties was used. The control group consisted of 50 selected patients $(62,50 \pm 5.54)$ with SR. The patients to undergo CV were performed Trans Esophageal Echocardiogram (TEE) using 5.0 Mhz TEE probe after sufficient fasting period and local anesthesia to exclude potential thrombus in Left Atrial Appendage (LAA), otherwise, if the patients rejected or could not tolerate TEE, they were anticoagulated with warfarin (target INR 2 -3) 3 to 4 weeks prior to CV. TEE was done for 74 patients and LAA flow rate was measured in centimeter per second $(\mathrm{cm} / \mathrm{s})$. CV was done with successful energy levels of 200,300,360, 360 joule monophasic wave shocks under midazolam $(0.05 \mathrm{mg} / \mathrm{kg}$ bolus $)$ anesthesia in the presence of its antidote (flumazenil). Amiodarone treatment was established in loading dose at least ten days prior to $\mathrm{CV}$ procedure. If the procedure was done immediately, amiodarone was given in a loading dose of $1200 \mathrm{mg} /$ day via IV route for 24 hours followed by $200 \mathrm{mg}$ daily. The patients performed TEE continued anticoagulation with warfarin for at least three weeks after the procedure regardless of whether they recovered sinus rhythm. Regarding the risk factors, some patients also received acetylsalicylic acid if there was no bleeding or intolerance.

The patients who had coronary artery disease, moderate-severe valvular heart disease, collagen tissue disorders, chronic infection, or fever were not allowed to participate in the study.

The patients were evaluated with anamnesis, physical examination, electrocardiography, thyroid function tests, routine laboratory blood analyses along with CRP, erythrocyte sedimentation rate, AntiThrombin 3 (AT3), D-dimer, fibrinogen, anti-cardiolipin antibodies. D-dimer was analyzed with immunoturbidimetric method; CRP was analyzed in serum using nephelometric method; AT3 was analyzed

*Corresponding author: Mehmet Demir, Department of Cardiology, Bilecik State Hospital, Yaseminpark sit 4E D11 osmangazi 16100 Bursa, Turkey, Tel: +90 2242573328; E-mail: drmehmetmd@gmail.com

Received March 24, 2012; Accepted April 25, 2012; Published April 27, 2012

Citation: Özmen G, Demir M, Birdane A (2012) The Effects of Cardioversion on the Prothrombotic Markers and CRP in the Atrial Fibrillation. J Blood Lymph 2:104. doi:10.4172/2165-7831.1000104

Copyright: (c) 2012 Demir M. This is an open-access article distributed under the terms of the Creative Commons Attribution License, which permits unrestricted use, distribution, and reproduction in any medium, provided the original author and source are credited. 
in plasma using immunochemical assay; fibrinogen was analyzed with clotting method; anti-cardiolipin IgG and IgM were tested using Enzyme-Linked Immunosorbent Assay (ELISA).

Acuson (Sequia Siemens) device and $3.75 \mathrm{MHz}$ probe were used for transthoracic echo assessing the diameter of the left atrium, left ventricular ejection fraction, and other echo findings. The examination was done in the left lateral decubitus position. The measurements were done according to the recommendations established by the American Echocardiography Society. The left ventricular wall thickness, endsystolic and end-diastolic diameters were measured in parasternal long-axis M-mode image; the diameter of the left atrium was measured in parasternal long-axis view in millimeter. The ejection fraction was measured with "teicholtz" method or with modified Simpson and "area length" methods individually in the patients having abnormal segmental contraction. Doppler echo measurements were done in apical four-chamber view with sample volume positioned on the mitral leaflets. Transmitral early and late diastolic velocities were measured in $\mathrm{cm} / \mathrm{s}$ and recorded in $\mathrm{mmHg}$. The patients were evaluated for AF recurrence with ECG and blood samples and if possible, looking for mitral A wave on echo during follow-up visits at one day, one month, three months and six months.

\section{Statistical assessments}

For statistical analyses of the data achieved in the study, Statistical Package for the Social Sciences (SPSS) for Windows ver. 13.0 was used. Among numeric variables, those with normal distribution were analyzed with parametric tests, others not showing normal distribution were analyzed with non-parametric tests. The results were presented as mean \pm Standard Deviation (SD). Parametric tests were used for the variables with normal distribution; non-parametric tests were used for the variables not showing normal distribution.

\section{Results}

There were 57 (41\%) men and $82(59 \%)$ women among 89 AF patients and 50 control individuals with SR, who participated in the study; the mean age was $63,33 \pm 9.14$ in the AF group and $62.50 \pm 5.54$ in the control group. There was no significant difference between two populations in terms of age and gender. In both groups, Hemoglobin, hematocrit (Htc), WBC count, thrombocyte count, erythrocyte sedimentation rate, fibrinogen, CRP, D-dimer, anti-thrombin 3, anticardiolipin IgG and IgM levels were analyzed. When two groups were compared in terms of other risk factors, there was no difference between the groups in terms of diabetes, family history, hypertension and cigarette smoking. Left atrial dimension were higher in the AF group than controls (Table 1). All 89 patients in the AF group were performed electrical CV. After CV, 66 patients (74.1\%) returned to SR. It was unsuccessful in 23 patients. AF recurred in 36 patients (54.5\%) within six months. Seven patients died during follow-up, six of them were in the group of the patients who had AF recurrence.

CRP, fibrinogen, D-dimer and anti-thrombin 3 levels were found to be significantly higher in AF patients in comparison with healthy individuals $(\mathrm{p}<0.05)$ (Table 1). Basal CRP levels of 30 patients who had relapses in six months and CRP levels of the patients maintaining SR were compared; although CRP level was slightly higher in the patients with recurrent $\mathrm{AF}$, the difference was not statistically significant $(\mathrm{p}=0.62)$ (Table 2).

The relationship between prothrombotic markers levels and LAA velocity and LA SEC status could not be determined (Table 3-5).

\begin{tabular}{|l|c|c|c|}
\hline & $\mathrm{AF}(\mathrm{n}=89)$ & Control $(\mathrm{n}=50)$ & $\mathrm{p}$ value \\
\hline age (years) & $63,33 \pm 9.14$ & $62,50 \pm 5.54$ & $\mathrm{NS}$ \\
\hline Males (n,\%) & $49(55 \%)$ & $32(64 \%)$ & $\mathrm{NS}$ \\
\hline Body massindex (kg/m2) & $22,12 \pm 2,15$ & $23,16 \pm 2,29$ & $\mathrm{NS}$ \\
\hline creatinin (mg/dl) & $0.98 \pm 0.32$ & $0.85 \pm 0.29$ & $\mathrm{NS}$ \\
\hline Leftatrium (mm) & $46,2 \pm 11$ & $35,2 \pm 9$ & $<0,05$ \\
\hline LV EF (\%) & $58,1 \pm 13$ & $63,4 \pm 15$ & $\mathrm{NS}$ \\
\hline TSH (mclU/ml) & $1.40 \pm 1.62$ & $1.26 \pm 1.51$ & $\mathrm{NS}$ \\
\hline Hypertension (n) & 22 & 23 & $\mathrm{NS}$ \\
\hline Diabetesmellitus (n) & 3 & 4 & $\mathrm{NS}$ \\
\hline Smoking (n) & 8 & 9 & $\mathrm{NS}$ \\
\hline Hematokrit (\%) & $40,76 \pm 2,53$ & $41,29 \pm 3,30$ & $\mathrm{NS}$ \\
\hline Leukosit (U/L) & $8000 \pm 14,23$ & $6700 \pm 6,35$ & $<0,05$ \\
\hline Platelet (U/L)x1000 & $230 \pm 8,67$ & $209 \pm 6,78$ & $\mathrm{NS}$ \\
\hline INR & $1,20 \pm 0,29$ & $1,02 \pm 0,23$ & $<0,05$ \\
\hline CRP (mg/dl) & $0,58 \pm 0,12$ & $0,32 \pm 0,1$ & $<0,05$ \\
\hline Antitrombin-3 (mg/dl) & $27,43 \pm 7,03$ & $29,98 \pm 8,95$ & $<0,05$ \\
\hline ESR (mm/heur) & $15,5 \pm 3,61$ & $11 \pm 2,35$ & $\mathrm{NS}$ \\
\hline Fibrinogen (mg/dl) & $406,5 \pm 98,2$ & $371 \pm 87,3$ & $<0,05$ \\
\hline D- dimer (mg/dl) & $0,43 \pm 0,11$ & $0,16 \pm 0,1$ & $\mathrm{NS}$ \\
\hline AnticardiolipinlgG & $8,25 \pm 4,46$ & $7,60 \pm 3,78$ & $\mathrm{NS}$ \\
\hline AnticardiolipinlgM & $6,75 \pm 2,96$ & $5,95 \pm 2,54$ & $\mathrm{NS}$ \\
\hline
\end{tabular}

Table 1: Baseline caracteristicof AFpatientsandcontrolsgroup.

\begin{tabular}{|c|c|c|c|}
\hline & Maintaining SR $(n=30)$ & Recurrent AF $(n=30)$ & $P$ \\
\hline $\operatorname{CRP}(\mathrm{mg} / \mathrm{dl})$ & $0,220,09$ & $0,400,11$ & 0,62 \\
\hline
\end{tabular}

Table 2: Comparison of basal CRP levels in patientwithmaintaining SR andrerecurrent AF.

\begin{tabular}{|c|c|c|c|c|c|c|}
\hline & & $\mathbf{n}$ & \multicolumn{3}{|c|}{ Persentil } & \multirow{2}{*}{ p } \\
\cline { 3 - 6 } & & & Median & $\mathbf{2 5}$ & $\mathbf{7 5}$ & \\
\hline $\begin{array}{c}\text { LAA } \\
\text { emptyingvelocity } \\
(\mathrm{cm} / \mathrm{sn})\end{array}$ & SR & 24 & 28,15 & 20,00 & 34,25 & NS \\
\hline
\end{tabular}

Table 3: Comparisonof LAAemptyingvelocityof AF versussinusrhytm at 6.month.

\begin{tabular}{|l|c|c|c|}
\hline & $\begin{array}{c}\text { LAAvelocity } \\
<21(\mathrm{~cm} / \mathrm{sn})\end{array}$ & $\begin{array}{c}\text { LAA velocity } \\
>21\end{array}$ & $\mathrm{P}$ \\
\hline Antitrombin-3(mg/dl) & $26,54 \pm 7,5$ & $27,40 \pm 6,5$ & $\mathrm{NS}$ \\
\hline Fibrinogen & $403 \pm 122$ & $395 \pm 116$ & $\mathrm{NS}$ \\
\hline CRP(mg/dl) & $0,55 \pm 0,51$ & $0,46 \pm 0,23$ & $\mathrm{NS}$ \\
\hline anticardiolipinlgG & $7,10 \pm 1,6$ & $9,10 \pm 2,3$ & $\mathrm{NS}$ \\
\hline anticardiolipinlgM & $6,55 \pm 1,99$ & $6,90 \pm 2,1$ & $\mathrm{NS}$ \\
\hline D-dimer(mg/dl) & $0,66 \pm 0,32$ & $0,31 \pm 0,15$ & $\mathrm{NS}$ \\
\hline
\end{tabular}

Table 4: The relationship between LAA emptying velocity and prothromboticmark ers.

\begin{tabular}{|l|c|c|c|}
\hline & SEC (no /mild) & SEC (severe) & $\mathrm{p}$ \\
\hline Antitrombin-3 (mg/dl) & $26,24 \pm 7,80$ & $29,00 \pm 5,41$ & $\mathrm{NS}$ \\
\hline CRP(mg/dl) & $0,37 \pm 0,13$ & $0,80 \pm 0,25$ & $\mathrm{NS}$ \\
\hline anticardiolipinlgG & $8,5 \pm 1,6$ & $7,95 \pm 2,14$ & $\mathrm{NS}$ \\
\hline anticardiolipinlgM & $6,25 \pm 2,1$ & $0,29 \pm 0,12$ & $\mathrm{NS}$ \\
\hline D-dimer & $0,35 \pm 0,13$ & $0,42 \pm 0,26$ & $\mathrm{NS}$ \\
\hline Fibrinogen & $391,5 \pm 12,8$ & $480 \pm 23,6$ & $\mathrm{NS}$ \\
\hline
\end{tabular}

Table 5: The relationship between Leftatrial SEC and prothromboticmarkers.

The median level of CRP was $0.22 \mathrm{mg} / \mathrm{dl}$ in the patients maintaining SR at the end of six months, whilst this value was $0.48 \mathrm{mg} / \mathrm{dl}$ in the patients with AF recurrences and permanent AF $\mathrm{p}<0.05$ ) (Table 6, Figure 1)

The initial median CRP level $(0.54 \mathrm{mg} / \mathrm{dl})$ decreased to $0.22 \mathrm{mg} / \mathrm{dl}$ in the patients maintaining SR Whereas in the recurrent AF group CRP higher than basal at six month but was statistically nonsignificant $(0.22$ 


\begin{tabular}{|l|l|c|c|c|c|c|}
\hline & & \multirow{2}{*}{$\mathbf{n}$} & \multicolumn{3}{|c|}{ Persentil } & \multirow{2}{*}{$\mathbf{P}$} \\
\cline { 4 - 7 } & & & Median & $\mathbf{2 5}$ & $\mathbf{7 5}$ & \\
\hline CRP & SR & 30 & 0,22 & 0,11 & 0,55 & $<0,05$ \\
(mg/dl) & AF (recurrent+permenant) & 52 & 0,48 & 0,23 & 0,88 & \\
\hline
\end{tabular}

Table 6: (6. Month) maintaining SR patientsandrecurrentandpermenant AF patients.

\section{SR and CRP}

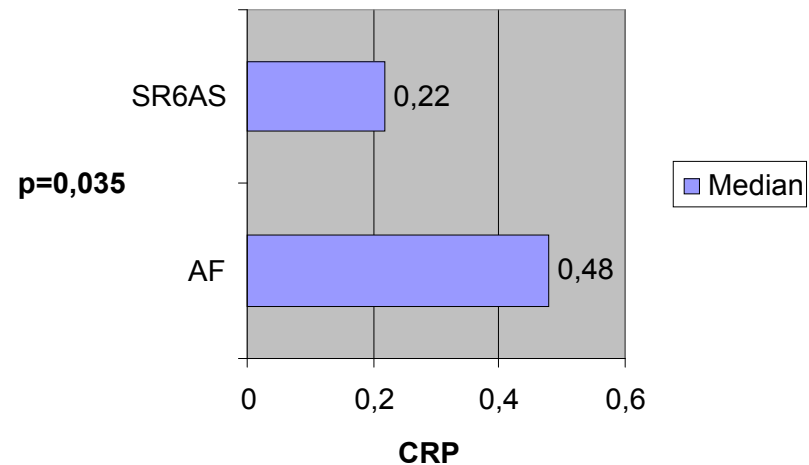

Figure 1: CRP level in patientswithmaintaining SR and recurrentpluspermenan AF.

vs $0.40 \mathrm{mg} / \mathrm{dl})$ (Table 7).

When we compared of patients for whom CV procedure was successful and remaining SR and 23 patients for whom CV failed, the median CRP levels were $0.22 \mathrm{mg} / \mathrm{dl}$ and $0.67 \mathrm{mg} / \mathrm{dl}$, respectively ( $\mathrm{p}<$ 0.05) (Table 8, Figure 2).

LA dimension were higher in patients with maintain in SR than recurrent and permanent $\mathrm{AF}$ patients at six month (Table 9).

The median level of D-dimer raised from $0.175 \mu \mathrm{g} / \mathrm{dl}$ (before CV) to $0.29 \mu \mathrm{g} / \mathrm{dl}$ (six months after CV) in the patients who were performed CV successfully and maintained SR. However, we did not observed similar situation in fibrinogen, anti-thrombin 3, anti-cardiolipin levels (Table 10).

\section{Discussion}

Chronic symptoms due to AF impair general well-being and quality of life. Additionally, severe complications such as systemic embolism, hemodynamic dysfunction resulting in congestive heart failure, cardiomyopathy may occur in these patients. Treatment regimens given to prevent these complications are long-term and costly. Antiarrhythmic drugs are not efficacious in every patient or cannot be used in certain situations because of their side effects. Recently, several guidelines and algorithm-based treatment recommendations have been published to choice optimum treatment option for AF. However, it is obvious that a general approach cannot be suitable for all patients having AF, which is a complex arrhythmia.

If an appropriate rhythm control approach and CV are chosen and performed accurately in AF patients, SR can be restored in $70-90 \%$ of the patients, although several well-known factors such as duration of arrhythmia, diameter of the left atrium, underlying reason of AF, which affect the success of $\mathrm{CV}$ have an impact on the outcome. AF recurs in approximately $53 \%$ of these patients in months following successful cardioversion, and only 23\% of the patients maintain SR over 5 years despite of appropriate antiarrhythmic therapy. In our study, SR success

\begin{tabular}{|l|c|c|c|}
\hline & Basal CRP $(\mathbf{m g} / \mathbf{d l})$ & $\mathbf{6 . m o n t h}$ CRP $(\mathbf{m g} / \mathbf{d l})$ & $\mathbf{P}$ \\
\hline SR $(n=30)$ & $0,54 \pm 0,23$ & $0,22 \pm 0,15$ & $<0,05$ \\
\hline Recurrent AF $(n=30)$ & $0,22 \pm 0,09$ & $0,40 \pm 0,11$ & NS \\
\hline
\end{tabular}

Table 7: Basaland 6. Month CRP levels inpatientswithmaintaining SR andrecurrent AF.

\begin{tabular}{|l|l|c|c|c|c|c|}
\hline & & \multirow{2}{*}{$\mathbf{n}$} & \multicolumn{3}{|c|}{ Persentil } & \multirow{2}{*}{ p } \\
\cline { 4 - 7 } & & & Median & $\mathbf{2 5}$ & $\mathbf{7 5}$ & \\
\hline \multirow{2}{*}{ CRP (mg/dl) } & 6. month SR & 30 & 0,22 & 0,11 & 0,55 & \multirow{2}{*}{ ) } \\
\cline { 4 - 6 } & Permenant AF & 23 & 0,67 & 0,28 & 0,98 & \\
\hline
\end{tabular}

Table 8: CRP level in patientswithmaintainingSR andpermenant AF (CV failed) (6.month), Mann - Whitney U Test.

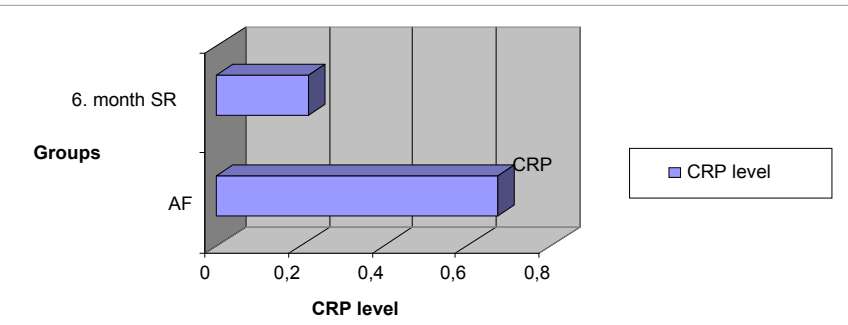

Figure 2: CRP level in patientswithmaintaining SR and permenant AF (CV failed) (6.month).

\begin{tabular}{|l|c|c|c|}
\hline & 6. month SR $\mathbf{n = 3 0}$ & $\begin{array}{c}\text { 6.month AF (recurrent+ per- } \\
\text { menant) } \mathbf{n = 5 2}\end{array}$ & $\mathbf{p}$ \\
\hline Leftatrium $(\mathrm{mm})$ & $44,31 \pm 6,2$ & $48,23 \pm 6,3$ & $<0,05$ \\
\hline
\end{tabular}

Table 9: Comparison of LA dimensions of thepatientswithmaintaining SR andrecurrentandpermenant $\mathrm{AF}$

\begin{tabular}{|l|c|c|c|}
\hline & Basal & $\mathbf{6 . m o n t h}$ & P \\
\hline Fibrinogen & $413,13 \pm 113,51$ & $392,37 \pm 98,05$ & NS \\
\hline Antithrombin- 3 & $28,79 \pm 5,7$ & $30,08 \pm 5,8$ & NS \\
\hline D- dimer & $0,175 \pm 0,11$ & $0,29 \pm 0,22$ & NS \\
\hline AnticardiolipinlgG & $9,2 \pm 1,36$ & $5,2 \pm 1,13$ & NS \\
\hline AnticardiolipinlgM & $6,4 \pm 1,58$ & $6 \pm 1,5$ & NS \\
\hline
\end{tabular}

Table 10: Basaland 6.month prothromboticmarkers in patientwithmaintaining SR.

after CV was $74.8 \%$; sinus rhythm could not be restored in only 23 patients (25.2\%). AF relapsed in 36 patients (54.5\%) within six months; at the end of six months, only 30 patients (nearly 33\%) maintained SR. The well-known reason of unpromising outcomes is electrical and structural remodeling. This situation causes progressive enlargement of LA and atrial fibrosis, loss of regular velocity, and rapid atrial signal transduction. It is now accepted the opinion of that these changes ensure the persistence of arrhythmia in the atrium. It is controversial whether inflammation has an impact on triggering and maintenance of AF. However, the data achieved in the studies suggest that inflammation is of importance in this pathologic pathway. Conway Dwayne S.G. et al. [15] researched the effects of inflammation and coagulation system on restoration of SR by CV in AF cases. In that study, there was no association with coagulation system, endothelial function, thrombocyte activation at the time of $\mathrm{CV}$ procedure and two months later, whilst sensitive CRP (hsCRP) levels above median values was related to poor CV success and in multivariate analysis, it was found to be an independent indicator of CV success [15]. In that study, CRP levels were found to be significantly high in AF patients in comparison with healthy individuals $(\mathrm{p}<0.05)$, however, the other prothrombotic markers did not differ. In our study, basal levels of CRP were found to be remarkably higher in the AF group in comparison with the individuals with SR $(p<0.05)$, additionally, median levels of prothrombotic markers such 
as D-dimer, fibrinogen, anti-thrombin 3 were statistically significantly higher in AF cases. When we compared the subgroup of patients for whom CV procedure was successful and remaining SR and 23 patients for whom CV failed, the median CRP levels were $0.22 \mathrm{mg} / \mathrm{dl}$ and 0.67 $\mathrm{mg} / \mathrm{dl}$, respectively $(\mathrm{p}<0.05)$ (Table 7 , Figure 2 ). Basal CRP levels of 30 patients who had relapses in six months and CRP levels of the patients maintaining SR were compared; although CRP level was slightly higher in the patients with recurrent AF, the difference was not statistically significant. In our study, the diameter of SA was significantly greater in the patients for whom CV failed. CRP was analyzed with nephelometric method. We think that high-sensitivity CRP (hsCRP) analysis could have generated results more precise. There was no difference between two groups in terms of gender, diabetes (Diabetes Mellitus), cigarette smoking, heredity, Hypertension (HT) and dyslipidemia. There was no correlation between $\mathrm{CV}$ and the LAA flow rate with spontaneous echo contrast (SEC) in the left atrium. There was no relationship between SEC density and prothrombotic markers.

Several hypotheses about the causative relationship between inflammation and AF have been proposed and there are different results in the literature. It is a well-known truth disclosed by many studies that there are prothrombotic state and tendency to thromboembolism in AF. The question of whether prothrombotic state remains after restoration of sinus rhythm attracts many researchers' attention. There are studies conducted with the patients taking anticoagulant agents and those not using such medicines; the level of fibrin D-dimer, which is an indicator of prothrombotic state in the body, is found to be lower in the patients receiving this treatment. It is also interesting the question of whether existent prothrombotic state regresses with long-term maintenance of SR or whether prothrombotic state persists despite of the restoration of SR.

In our study, the median level of CRP was $0.22 \mathrm{mg} / \mathrm{dl}$ in the patients maintaining SR at the end of six months, whilst this value was $0.40 \mathrm{mg} /$ $\mathrm{dl}$ in the patients with AF recurrences; however, the difference was not statistically significant $(\mathrm{p}=0.06)$. In two recent studies, found that lower basal CRP levels were related to more often short- and long-term maintenance of sinus rhythm after CV [16,17]. However, Tveit et al. [18] did not find a decline in the levels of inflammatory markers of CRP and IL- 6 during six-month maintenance of sinus rhythm. This result suggests that inflammatory state in AF patients should not be affected by SR restoration with CV. In contrast, Climent et al. [19] achieved a significant decline comparing the values of the patients maintaining SR one month after $C V$ with pre-CV values $(p=0.03)$. Our results are consistent with the results reported by Climent et al. [19]. The initial median CRP level $(0.54 \mathrm{mg} / \mathrm{dl})$ decreased to $0.22 \mathrm{mg} / \mathrm{dl}$ in the patients maintaining SR. In contrast to the meta-analysis performed by Tveit et al. this data asserts that AF or co-morbid factors generate inflammatory state in these patients and inflammation is ameliorated by restoration and maintenance of sinus rhythm with CV [18]. In our study, the median CRP level was $0.32 \mathrm{mg} / \mathrm{dl}$ in the patients with SR (the control group) and in the AF group, CRP level analyzed before CV was $0.58 \mathrm{mg} / \mathrm{dl}$; this difference was statistically significant $(\mathrm{p}<0.001)$. The median level of CRP was $0.22 \mathrm{mg} / \mathrm{dl}$ in the patients maintaining SR at the end of six months, whilst median level of CRP was $0.48 \mathrm{mg} / \mathrm{dl}$ in the patients for whom CV failed initially or who responded successfully to $\mathrm{CV}$ but subsequently returned to AF; this difference was also statistically significant $(\mathrm{p}<0.05)$. That the median level of CRP in our study was $0.22 \mathrm{mg} / \mathrm{dl}$ in the patients maintaining SR but $0.67 \mathrm{mg} / \mathrm{dl}$ in the patients for whom CV failed $(\mathrm{p}<0.05)$ suggests that inflammation can be reduced by restoration and maintenance of SR
Many studies researched the probability of an accompanying hypercoagulable state that may contribute development of thromboembolism in atrial fibrillation. In a study, plasma prothrombin fragment, thrombin, anti-thrombin 3 levels were found to be higher in AF patients with mitral stenosis in comparison with those without MS $[20,21]$. In a study performed to ascertain the hypothesis of that CV might increase prothrombotic state, Jacob K. et al. [22] did not find any difference in any time plasma median $\mathrm{D}$-dimer and protein $\mathrm{C}$ levels, however, the authors demonstrated a significant decline in plasma fibrinogen and anti-thrombin 3 levels in early period following $\mathrm{CV}$; this result indicates the formation of thrombin. This study asserts hemostatic changes occurring in thrombogenesis induced by cardioversion despite of warfarin treatment given to the patients [22]. Hypercoagulable state establishes by the conversion of atrial fibrillation into sinus rhythm with CV and the changes in plasma fibrin D-dimer levels have been demonstrated in many studies. From this point of view, prothrombotic markers are useful to predict the blood coagulation status and the changes usually occur immediately before CV and within hours and days after CV. In a study performed by Lip GY et al., after dividing the patients into two groups (the group 1 consisted of 7 patients given heparin within 24 hours prior to CV and the group 2 consisted of 12 patients previously given efficient anticoagulation), the authors analyzed D-dimer and fibrinogen levels before CV and 3, 7, and 14 days after CV. The authors did not find any difference between two groups in terms of fibrinogen levels; D-dimer levels decreased gradually up to day 14 in the group 1 in comparison with pre-CV levels, but did not differ in the group 2 probably due to the effect of anticoagulation. Basal D-dimer levels were found to be lower in the second group. In our study, we aimed to recognize the changes analyzing prothrombotic markers before $\mathrm{CV}$ and on the sixth month rather than within hours and days after successful CV. The fibrinogen and D-dimer levels were significantly higher in AF patients in comparison with the control group with sinus rhythm $(\mathrm{p}<0.05)$. The median level of $\mathrm{D}$-dimer raised from $0.175 \mu \mathrm{g} / \mathrm{dl}$ (before $\mathrm{CV}$ ) to $0.29 \mu \mathrm{g} / \mathrm{dl}$ (six months after $\mathrm{CV}$ ) in the patients who were performed CV successfully and maintained SR; this situation suggests the persistence of thrombotic process despite of the maintenance of SR. However, we did not observed similar situation in fibrinogen, anti-thrombin 3, anti-cardiolipin levels, there were only statistically insignificant changes.

Recently, numerous studies have been conducted about the causative relationship between inflammation, thrombotic state and AF. That CRP level also peaks at 48-72 hours after by-pass surgery when AF most often occurs suggests the association of AF with inflammation. In our study, basal CRP levels were found to be lower in the patients maintaining SR, although there was no statistically significant difference. The fact that CRP level was significantly lower in the patients maintaining SR at the end of six months in comparison with the patients for whom CV failed and the decline observed in the patients maintaining SR in comparison with basal CRP level may suggest that AF recurrence is less frequent among the patients with low CRP levels. The decrease in CRP levels after restoration of sinus rhythm affirms reduced inflammation. Therefore, it can be concluded that inflammation is the consequence of atrial fibrillation rather than being its cause.

The thrombotic state is associated with AF and accounts for thromboembolic events; in our study, increased D-dimer levels in the patients maintaining SR after six months suggests persisting prothrombotic state and the risk of embolic events.

The limitations of our study are small patient series and the lack of the data regarding the changes in prothrombotic markers in the 
Citation: Özmen G, Demir M, Birdane A (2012) The Effects of Cardioversion on the Prothrombotic Markers and CRP in the Atrial Fibrillation. J Blood Lymph 2:104. doi:10.4172/2165-7831.1000104

patients receiving anticoagulant agents since we did not formed a group of patient in terms of anticoagulant use among the patients maintaining SR. Another limitation is unavailability of high-sensitivity CRP (hsCRP) measurements.

\section{References}

1. Nattel S (2002) New ideas about atrial fibrillation 50 years on. Nature 415 219-226

2. Korantzopoulos P, Kolettis TM, Goudevenos JA, Siogas K (2005) Errors and pitfalls in the non-invasive management of atrial fibrillation. Int $\mathrm{J}$ Cardiol 104: 125-130.

3. Van Gelder IC, Hemels ME (2006) The progressive nature of atrial fibrillation: a rationale for early restoration and maintenance of sinus rhythm. Europace 8: 943-949.

4. Chung MK (2004) Randomized trials of rate vs. rhythm control for atria fibrillation. J Interv Card Electrophysiol 10: 43-53.

5. Korantzopoulos P, Kolettis T, Siogas K, Goudevenos J (2003) Atrial fibrillation and electrical remodeling: the potential role of inflammation and oxidative stress. Med Sci Monit 9: RA225-RA229.

6. Hernández Madrid A (2006) C-reactive protein and atrial fibrillation. An old marker looking for a new target. Rev Esp Cardiol 59: 94-98.

7. Wazni O, Martin DO, Marrouche NF, Shaaraoui M, Chung MK, et al. (2005) C reactive protein concentration and recurrence of atrial fibrillation after electrical cardioversion. Heart 91: 1303-1305.

8. Malouf JF, Kanagala R, Al Atawi FO, Rosales AG, Davison DE, et al. (2005) High sensitivity C-reactive protein: a novel predictor for recurrence of atrial fibrillation after successful cardioversion. J Am Coll Cardiol 46: 1284 -1287.

9. Watanabe E, Arakawa T, Uchiyama T, Kodama I, Hishida H (2006) Highsensitivity $\mathrm{C}$-reactive protein is predictive of successful cardioversion for atrial fibrillation and maintenance of sinus rhythm after conversion. Int J Cardiol 108: 346-353

10. Lip GY (1995) Does atrial fibrillation confer a hypercoagulable state? Lancet 346: 1313-1314.

11. Kumagai K, Fukunami M, Ohmori M, Kitabatake A, Kamada T, et al. (1990) Increased intracardiovascular clotting in patients with chronic atrial fibrillation. $J$ Am Coll Cardiol 16: 377-380.

12. Lip GY, Lowe GD, Rumley A, Dunn FG (1996) Fibrinogen and fibrin D-dimer levels in paroxysmal atrial fibrillation: evidence for intermediate elevated levels of intravascular thrombogenesis. Am Heart J 131: 724-730.

13. Heppell RM, Berkin KE, McLenachan JM, Davies JA (1997) Haemostatic and haemodynamic abnormalities associated with left atrial thrombosis in nonrheumatic atrial fibrillation. Heart 77: 407-411.

14. Goldman ME, Pearce LA, Hart RG, Zabalgoitia M, Asinger RW, et al. (1999) Pathophysiologic correlates of thromboembolism in nonvalvular atrial fibrillation: I. Reduced flow velocity in the left atrial appendage (The Stroke Prevention in Atrial fibrillation [SPAF-III] study). J Am Soc Echocardiogr 12: 1080-1087.

15. Conway DS, Buggins P, Hughes E, Lip GY (2004) Predictive value of indexes of inflammation and hypercoagulability on success of cardioversion of persistent atrial fibrillation. Am J Cardiol 94: 508-510.

16. Gosselink ATM, Crijns HJGM, Van Gelder IC, Hans Hillige, Wiesfeld ACP et al. (1992) Low-dose amiodarone for maintenance of sinus rhythm after cardioversion of atrial fibrillation or flutter. JAMA 267: 3289-3293.

17. Psychari SN, Apostolou TS, Sinos L, Hamodraka E, Liakos G, et al. (2005) Relation of elevated C-reactive protein and interleukin-6 levels to left atrial size and duration of episodes in patients with atrial fibrillation. Am J Cardiol 95: 764-767.

18. Tveit A, Seljeflot I, Grundvold I, Abdelnoor M, Smith P, et al. (2007) Effect of candesartan and various inflamatory markers on maintenance of sinus rhytm after electrical cardioversion for atrial fibrillation. Am J Cardiol 99: 1544-1548.

19. Climent V, Marín F, Mainar L, Roldán V, García A, et al. (2009) Influence of electrical cardioversion on inflammation and indexes of structural remodeling, in persistent atrial fibrillation. Int J Cardiol 132: 227-232.

20. Asakura H, Hifumi S, Jokaji H, Saito M, Kumabashiri I, et al. (1992) Prothrombin fragment F1+2 and thrombin-antithrombin III complex are useful markers of the hypercoagulable state in atrial fibrillation. Blood Coagul Fibrinolysis 3: 469-473.

21. Gustafsson C, Blomback M, Britton M, Hamsten A, Svensson J (1990) Coagulation factors and the increased risk of stroke in nonvalvular atrial fibrillation. Stroke 21: 47-51.

22. Jacob K, Talwar S, Copplestone A, Gilbert TJ, Haywood GA (2004) Activation of coagulation occurs after electrical cardioversion in patients with chronic atrial fibrillation despite optimal anticoagulation with warfarin. Int J Cardiol 95: 83-88.

23. Lip GY, Rumley A, Dunn FG, Lowe GD (1995) Plasma fibrinogen end fibrin $\mathrm{D}$-dimer in patients with atrial fibrillation: effects of cardioversion to sinus rhythm. Int J Cardiol 51: 245-251. 